\title{
ON OPTIMAL EXTRUSION DIES FOR RIGID-PLASTIC MATERIALS
}

\author{
BY \\ HANS F. WEINBERGER \\ School of Mathematics, University of Minnesota \\ To my friend George Handelman
}

1. Introduction. In 1962 Richmond and Devenpeck [3] raised the question of how to design an extrusion die in such a way that the power that must be exerted to convert a bar of a prescribed cross section to a product of prescribed cross section at a prescribed speed is as small as possible. They showed that if one models the problem as a rigidplastic flow with the Tresca yield condition, this problem can be solved in the case of strip drawing, in which there is no change in a transverse direction. Richmond and Morrison [4] extended this result to wire-drawing, in which case the die and the flow are axially symmetric. In both of these cases, the optimality condition turned out to be that the velocity vector is an eigenvector corresponding to the largest eigenvalue of the rate-of-strain matrix, and the authors called such a flow a streamlined flow.

It was pointed out by Hill [1] that the concept of streamlined flow (which he called ideal flow) can be extended to three dimensions, and he gave a heuristic argument to indicate that any optimal die should produce a flow with this property.

In lectures at the Seminar on Industrial Problems of the Institute for Mathematics and its Applications, Owen Richmond and Russ Mallett aroused the author's interest in the problem of computing optimal dies that produce general shapes such as I-beams.

One can approach this problem in two ways. One is to assume that Hill's argument is correct, and to seek a good algorithm to compute a streamlined flow that starts with a prescribed ingot cross section and ends with a prescribed product cross section. Some remarks on this approach are to be found in [5], but they did not lead to a satisfactory solution.

The purpose of the present work is to show that while optimization of a twodimensional or axially symmetric die leads to a streamlined flow, the same is not true for more general dies. Consequently, one must search among a more general class of flows in order to optimize the extrusion of a beam of prescribed cross section. 
2. The Tresca variational law. Let $\mathbf{v}(\mathbf{x})$ be a steady velocity field in three-space. The corresponding rate-of-strain matrix is

$$
D_{i j}=\frac{1}{2}\left(v_{i, j}+v_{j, i}\right) .
$$

(We use the symbol ${ }_{, i}$ to denote partial differentiation.)

We shall model the flow through the die as rigid-plastic flow. That is, there is a prescribed closed convex set $S$ in the space of symmetric $3 \times 3$ matrices with the property that the stress $\sigma$ is constrained to lie in this set, and that when $\sigma$ is in the interior of $S$, the rate of strain $D=0$, so that the flow at such points is a rigid-body flow.

We shall assume that the remaining flow laws can be obtained from a variational principle in the following manner. For each symmetric matrix $D$ define the support function

$$
H(D)=\sup _{\sigma \in S} \operatorname{tr}(\sigma D)
$$

Then the velocity field is the vector field that minimizes

$$
\int H(D) d \mathbf{x}
$$

where the integral is taken over the material that is being processed (see Fig. 1), and $\mathbf{v}$ is required to be tangential on the die boundary and the lateral boundaries of the material, and to match prescribed constant velocities and cross sections on the upstream and downstream sides. (Because $H(D)=0$ where the material is rigid, the integration is actually over a finite set.)

The equations of motion are the Euler equations of this variational problem.

We shall consider the Tresca yield criterion, which states that the shear stress on every plane is to be bounded by a positive material constant $k$. Then

$$
S=\left\{\sigma:|\mathbf{n}|^{2}|\sigma \mathbf{n}|^{2}-(\mathbf{n} \cdot \sigma \mathbf{n})^{2} \leq k^{2}|\mathbf{n}|^{4} \quad \text { for all } \mathbf{n}\right\} .
$$

It is easily checked that in this case

$$
H(D)= \begin{cases}2 k\|D\|_{l_{2}} & \text { when } \operatorname{tr}(D)=0 \\ +\infty & \text { when } \operatorname{tr}(D) \neq 0\end{cases}
$$

where $\|D\|_{l_{2}}$ denotes the norm of the linear transformation $D$ on the Euclidean space $l_{2}$. The reason for the second line is that if $\sigma$ lies in $S$, so does $\sigma-p I$ for all values of $p$. The effect is that a minimizer of (2.2) must satisfy the constraint $\operatorname{tr}(D)=0$. That is, $\operatorname{div}(\mathbf{v})=0$, so that the flow is incompressible.

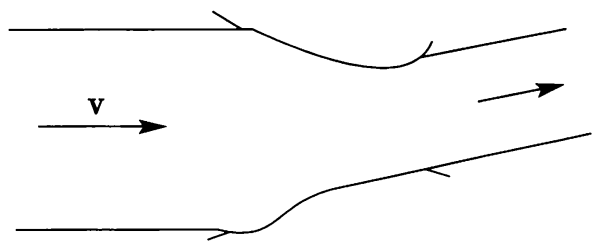


The Euler equation for the resulting constrained minimum is most easily written in the following form. The stress is given by

$$
\sigma_{i j}=\frac{\partial H(D)}{\partial D_{i j}}-p \delta_{i j}
$$

where $p$ is a Lagrange multiplier. The equilibrium equations are

$$
\sigma_{i j, j}=0 \text {. }
$$

(We shall use the summation convention.) In addition, we have the natural boundary condition

$$
\mathbf{n} \times(\sigma \mathbf{n})=0
$$

on the die and lateral boundaries. This condition states that the boundaries are "well lubricated".

We recall that for the Tresca law, $H(D):=2 k\|D\|_{l_{2}}$, where $\|D\|_{l_{2}}$ is the norm of the linear transformation $D$ on the Euclidean space $l_{2}$. Because $D$ is symmetric, this norm is the largest of the absolute values of the eigenvalues of $D$. Let $\mathbf{q}$ be an eigenvector that corresponds to this eigenvalue. We suppose that the determinant of $D$ is not zero. Then the eigenvalue of largest magnitude is uniquely defined, because $\operatorname{tr}(D)=0$. A computation shows that

$$
\frac{\partial\|D\|_{l_{2}}}{\partial D_{i j}}=\operatorname{sgn}(\lambda) \frac{q_{i} q_{j}}{\mid \mathbf{q}^{2}} .
$$

We see from (2.3) that the equilibrium conditions (2.4) can be written as

$$
\left(q_{i, j}+q_{j, i}\right) q_{j}-\frac{1}{|\mathbf{q}|^{2}} q_{k}\left(q_{k, l}+q_{l, k}\right) q_{l} q_{i}+q_{k, k} q_{i}=|\mathbf{q}|^{2}[\ln |\mathbf{q}|+\operatorname{sgn}(\lambda) p /(2 k)]_{, i}
$$

We now choose the otherwise undetermined magnitude

$$
|\mathbf{q}|=e^{-\operatorname{sgn}(\lambda) p /(2 k)},
$$

so that the right-hand side above is zero. Then the equilibrium condition becomes

$$
\left(q_{i, j}+q_{j, i}\right) q_{j}-\frac{1}{|\mathbf{q}|^{2}} q_{k}\left(q_{k, l}+q_{l, k}\right) q_{l} q_{i}+q_{k, k} q_{i}=0 .
$$

Because the second term on the left is the orthogonal projection of the first term in the direction of $\mathbf{q}$, this system is equivalent to the system

$$
\begin{aligned}
\left(q_{i, j}+q_{j, i}\right) q_{j}-\gamma q^{i} & =0 \\
\operatorname{div} \mathbf{q} & =0
\end{aligned}
$$

where $\gamma$ is a new scalar-valued unknown.

The same reasoning shows that the fact that $\mathbf{q}$ is an eigenvector of $D$ and the fact that $\operatorname{div}(\mathbf{v})=0$ are equivalent to the system

$$
\left(v_{i, j}+v_{j, i}\right) q_{j}-\frac{1}{|\mathbf{q}|^{2}} q_{k}\left(v_{k, l}+v_{l, k}\right) q_{l} q_{i}+v_{k, k} q_{i}=0 .
$$

Equations (2.6) and (2.7) are the equations of motion for a rigid-plastic material with the Tresca variational law. 
It is easily seen that the system (2.7) is hyperbolic. A surface is characteristic with respect to this system if either it is tangent to $\mathbf{q}$ or its normal makes a $45^{\circ}$ angle with $\mathbf{q}$. Because the system (2.6) is just (2.7) with $\mathbf{v}$ replaced by $\mathbf{q}$, it has the same characteristic surfaces. Therefore the whole system (2.6), (2.7) has these same characteristic surfaces, but each one has multiplicity 2 .

3. A sufficient condition for an optimal die. Because the function $H(D)=$ $2 k\|D\|_{l_{2}}$ is homogeneous of degree one, and because we only work with trace-free matrices $D$, we see from $(2.3)$ that $\operatorname{tr}(\sigma D)=H(D)$. Thus the integral of $H(D)$, which is minimized for a fixed die, gives the rate of dissipation, which is equal to the power that must be applied in order to force the material through the die. Richmond and Devenpeck defined a die to be optimal if this power is minimized.

In other words, an optimal die is one for which the integral in (2.2) is minimized among all dies that transform a prescribed upstream velocity and cross section to a prescribed downstream velocity and cross section, as well as among all divergence-free vector fields $\mathbf{v}$ that satisfy the upstream and downstream conditions and are tangent to each die boundary.

In order to obtain a sufficient condition for optimality, we observe that for any nonzero vector $\mathbf{v}$ and, in particular, for the velocity vector

$$
\frac{\mathbf{v} \cdot D \mathbf{v}}{|\mathbf{v}|^{2}} \leq\|D\|_{l_{2}}=H(D) /(2 k) \text {. }
$$

We also note that by the definition of $D$ and the fact that $\operatorname{div}(\mathbf{v})=0$

$$
\frac{\mathbf{v} \cdot D \mathbf{v}}{|\mathbf{v}|^{2}}=\operatorname{div}[(\ln |\mathbf{v}|) \mathbf{v}]
$$

Thus $H(D)$ is bounded below by a perfect divergence, and the divergence theorem shows that

$$
\int H(D) d \mathbf{x} \geq 2 k A_{\text {up }}\left|\mathbf{V}_{\text {up }}\right| \ln \left(A_{\text {up }} / A_{\text {down }}\right) \text {. }
$$

Here $A_{\text {up }}$ and $A_{\text {down }}$ are the areas of the upstream and downstream cross sections, and $\mathbf{V}_{\text {up }}$ is the prescribed upstream velocity. We have made use of the mass conservation law $A_{\text {up }}\left|\mathbf{V}_{\text {up }}\right|=A_{\text {down }}\left|\mathbf{V}_{\text {down }}\right|$.

The inequality (3.2) gives a lower bound for the integral to be minimized in terms of the prescribed data only, and is independent of the die shape. Thus if equality holds, the corresponding flow is produced by an optimal die. Equality in (3.2) will hold if and only if equality holds in (3.1) at every point. Because $\|D\|_{l_{2}}$ is equal to the largest absolute value of the eigenvalues of $D$, equality in (3.1) is valid if and only if at every point, $D$ has an eigenvalue of largest absolute value that is nonnegative, and $\mathbf{v}$ is a corresponding eigenvector.

Richmond and Devenpeck called a flow with this property a streamlined flow. We have thus shown that if the solution for a given die and given upstream and downstream conditions is a streamlined flow, then the die is optimal.

In terms of the principal eigenvector $\mathbf{q}$, the condition for streamlined flow is just $\mathbf{v}=\beta \mathbf{q}$ for some scalar-valued function $\beta$. In general, it seems rather unlikely that the 
resulting system of 4 unknowns can satisfy the 6 equations (2.6), (2.7). However, because the system (2.6) is just (2.7) with $\mathbf{v}$ replaced by $\mathbf{q}$, one can indeed satisfy both equations by solving only (2.6) and choosing $\beta$ to be a constant. This miracle was observed by Richmond and Devenpeck [3] in two dimensions, by Richmond and Morrison [4] for axially symmetric flows, and by Hill [1] in the general case.

Since $\beta$ is a constant, the equations for streamlined flow are just

$$
\left(v_{i, j}+v_{j, i}\right) v_{j}-\frac{1}{|\mathbf{v}|^{2}} v_{k}\left(v_{k, l}+v_{l, k}\right) v_{l} v_{i}+v_{k, k} v_{i}=0 .
$$

This system is defined even when $D=0$ so that $\mathbf{q}$ is undefined.

The system is hyperbolic with simple characteristics. A surface is characteristic if and only if either it is tangent to $\mathbf{v}$ or its normal makes a $45^{\circ}$ angle with $\mathbf{v}$.

4. A necessary condition for optimality. Suppose that the die has the property that it minimizes the integral of $H(D)$, not only among all divergence-free vector fields that satisfy the upstream and downstream conditions, but also among all dies that produce flows that satisfy this condition. We shall use the method of transplantation [2] to obtain a consequence of this fact, which is then a necessary condition for optimality.

Let $\mathbf{y}=\mathbf{y}(\mathbf{x}, \varepsilon)$ be a one-parameter family of smooth one-to-one transformations with $\mathbf{y}(\mathbf{x}, 0)=\mathbf{x}$, and define

$$
\eta_{i}(x)=\frac{\partial y_{i}}{\partial \varepsilon}(x, 0)
$$

Define the new vector field $\mathbf{V}(\mathbf{y}, \varepsilon)$ by

$$
V_{i}(\mathbf{y}(\mathbf{x}, \varepsilon), \varepsilon)=\frac{1}{\operatorname{det}(\partial \mathbf{y} / \partial \mathbf{x})} v_{k}(\mathbf{x}) \frac{\partial y_{i}}{\partial x_{k}} .
$$

A computation shows that

$$
\frac{\partial V_{i}}{\partial y_{j}}=\frac{1}{\operatorname{det}(\partial \mathbf{y} / \partial \mathbf{x})}\left\{\frac{\partial}{\partial x_{l}}\left(v_{k} \frac{\partial y_{i}}{\partial x_{k}}\right)-v_{k} \frac{\partial y_{i}}{\partial x_{k}} \frac{\partial^{2} y_{n}}{\partial x_{l} \partial x_{m}} \frac{\partial x_{m}}{\partial y_{n}}\right\} \frac{\partial x_{l}}{\partial y_{j}}
$$

In particular, we find by putting $j=i$ and summing that

$$
\sum \frac{\partial V_{i}}{\partial y_{i}}=\frac{1}{\operatorname{det}(\partial \mathbf{y} / \partial \mathbf{x})} \sum \frac{\partial v_{i}}{\partial x_{i}}
$$

Since $\mathbf{v}$ is divergence-free, the vector field $\mathbf{V}$ is divergence-free on the image of the domain under the mapping.

Let $D_{i j}(\mathbf{y}, \varepsilon)=(1 / 2)\left(\partial V_{i} / \partial y_{j}+\partial V_{i} / \partial y_{j}\right)$. We note that

$$
\int_{y(\Omega, \varepsilon)} H\left(D(y, \varepsilon) d y=\int_{\Omega} H(D(y(x, \varepsilon), \varepsilon) \operatorname{det}(\partial \mathbf{y} / \partial \mathbf{x})) d x .\right.
$$

It is obvious from the definitions of $\mathbf{y}(\mathbf{x}, \varepsilon)$ and of $\mathbf{V}$ that at $\varepsilon=0, \mathbf{V}(\mathbf{y}(\mathbf{x}, 0)), 0)=\mathbf{v}(\mathbf{x})$, and hence also $\partial V_{i} / \partial y_{j}=\partial v_{i} / \partial x_{j}$. Moreover, $\operatorname{det}(\partial \mathbf{y} / \partial \mathbf{x})=1$, and $\partial x_{i} / \partial \varepsilon=-\eta_{i}(x)$ at 
$\varepsilon=0$. Therefore

$$
\begin{aligned}
\frac{\partial}{\partial \varepsilon}\left[\frac{\partial V_{i}}{\partial y_{j}} \operatorname{det}(\partial y / \partial x)\right]_{\varepsilon=0} & =\frac{\partial}{\partial x_{j}}\left(v_{k} \frac{\partial \eta_{i}}{\partial x_{k}}\right)-\frac{\partial v_{i}}{\partial x_{k}} \frac{\partial \eta_{k}}{\partial x_{j}}-v_{i} \frac{\partial^{2} \eta_{k}}{\partial x_{j} \partial x_{k}} \\
& =\frac{\partial}{\partial x_{j}}\left(v_{k} \frac{\partial \eta_{i}}{\partial x_{k}}-\frac{\partial v_{i}}{\partial x_{k}} \eta_{k}-v_{i} \frac{\partial \eta_{k}}{\partial x_{k}}\right)+\frac{\partial}{\partial x_{k}}\left(\frac{\partial v_{i}}{\partial x_{j}} \eta_{k}\right) .
\end{aligned}
$$

Thus we see from the divergence theorem and the equilibrium equation that

$$
\begin{aligned}
\frac{d}{d \varepsilon}\left[\int H(D) d y\right]_{\varepsilon=0} & =\int \frac{\partial H}{\partial D_{i j}} \frac{\partial}{\partial \varepsilon}\left(\operatorname{det}(\partial \mathbf{y} / \partial \mathbf{x}) \frac{\partial V_{i}}{\partial y_{j}}\right) d x \\
& =\int \sigma_{i j} \frac{\partial}{\partial \varepsilon}\left(\operatorname{det}(\partial \mathbf{y} / \partial \mathbf{x}) \frac{\partial V_{i}}{\partial y_{j}}\right) d x \\
& =\int_{b d y}\left\{\sigma_{i j} n_{j}\left(v_{k} \frac{\partial \eta_{i}}{\partial x_{k}}-\frac{\partial v_{i}}{\partial x_{k}} \eta_{k}-v_{i} \frac{\partial \eta_{k}}{\partial x_{k}}\right)+\sigma_{i j} \frac{\partial v_{i}}{\partial x_{j}} \eta_{k} n_{k}\right\} d S \\
& -\int \eta_{k} \frac{\partial v_{i}}{\partial x_{j}} \frac{\partial \sigma_{i j}}{\partial x_{k}} d x .
\end{aligned}
$$

Because $S_{i j}:=\partial H / \partial D_{i j}$ is homogeneous of degree zero and $\operatorname{div} \mathbf{v}=0$, we see that

$$
\frac{\partial v_{i}}{\partial x_{j}} \frac{\partial \sigma_{i j}}{\partial x_{k}}=D_{i j} \frac{\partial S_{i j}}{\partial D_{l m}} \frac{\partial D_{l m}}{\partial x_{k}}=0,
$$

so that the last integral in (4.1) is zero.

We now look at the boundary integral in the next-to-the-last line. We shall assume that $\eta$ vanishes outside the domain where nonrigid flow occurs, so that this integral is over the lateral boundary. Here we know that $\sigma_{i j} n_{j}=\alpha n_{i}$ for some scalar-valued function $\alpha$, and that $\mathbf{v} \cdot \mathbf{n}=0$.

We observe that

$$
v_{k} \frac{\partial \eta_{i}}{\partial x_{k}}-\frac{\partial v_{i}}{\partial x_{k}} \eta_{k}-v_{i} \frac{\partial \eta_{k}}{\partial x_{k}}=[\operatorname{curl}(\eta \times \mathbf{v})]_{i}
$$

Thus the first term in the boundary integral becomes

$$
\begin{aligned}
\int_{b d y} \alpha \mathbf{n} \cdot \operatorname{curl}(\eta \times \mathbf{v}) d S & =\int_{b d y}[\mathbf{n} \cdot \operatorname{curl}(\alpha \eta \times \mathbf{v})-\mathbf{n} \cdot \nabla \alpha \times(\eta \times \mathbf{v})] d S \\
& =-\int_{b d y} \mathbf{v} \cdot \nabla \alpha \eta \cdot \mathbf{n} d S
\end{aligned}
$$

by the Stokes theorem.

Because of the equilibrium equation (2.4), $\operatorname{div}(\sigma \mathbf{v})=\sigma_{i j} \partial v_{i} / \partial x_{j}$. Thus (4.1) becomes

$$
\frac{d}{d \varepsilon}\left[\int H(D) d y\right]_{\varepsilon=0}=\int_{b d y} \operatorname{div}[-\alpha \mathbf{v}+\sigma \mathbf{v}] \eta \cdot \mathbf{n} d S .
$$

If the domain minimizes the dissipation, this integral must be zero for arbitrary boundary values of $\eta$ and we conclude that we must have

$$
\operatorname{div}[-\alpha \mathbf{v}+\sigma \mathbf{v}]=0 \text {. }
$$

We see from the formula (2.3) for $\sigma$ and the formula (2.5) that $\sigma \mathbf{n}=2 k \operatorname{sgn}(\lambda)$ $\left(\mathbf{q} \cdot \mathbf{n} /|\mathbf{q}|^{2}\right) \mathbf{q}-p \mathbf{n}$. Then the well-lubricated boundary condition shows that $\mathbf{q}$ is either 
normal or tangent to the boundary. In the former case, $\alpha=2 k \operatorname{sgn}(\lambda)-p$, while in the latter case $\alpha=-p$. In either case, we see that because $\operatorname{div}(\mathbf{v})=0, \operatorname{div}[-\alpha \mathbf{v}]=\operatorname{div}[p \mathbf{v}]$.

Thus in view of (2.3) and (2.5) the necessary condition becomes

$$
\operatorname{div}\left[\frac{\mathbf{v} \cdot \mathbf{q}}{|\mathbf{q}|^{2}} \mathbf{q}\right]=0
$$

on the boundary of an optimal die.

5. Two-dimensional and axially symmetric optimal dies. We now look at the special case of plane flow. That is, we suppose that $v_{3}=0$, and that the other two components are independent of $x^{3}$. In this case, $D$ has a zero eigenvalue and the other two eigenvalues are negatives of each other. Since we deal only with matrices that satisfy the constraints, the differentiation formula (2.5) is still valid, modulo a scalar matrix. Because the two eigenvalues have equal magnitude, we may take the principal eigenvector $\mathbf{q}$ to be in the tangent direction. In this case, $\mathbf{v}=\beta \mathbf{q}$ on the boundary, and, since $\operatorname{div} \mathbf{q}=\mathbf{0}$, the necessary condition (4.3) on the boundary becomes $\mathbf{q} \cdot \nabla \beta=0$. Thus $\beta$ is a constant.

The left-hand sides of the equations (2.6) and (2.7) with $i=3$ are just zero. By subtracting $\beta$ times (2.6) from (2.7), we obtain the system

$$
\begin{aligned}
\left(\begin{array}{cc}
\left(q_{1}^{2}+3 q_{2}^{2}\right) q_{1} & \left(q_{2}^{2}-q_{1}^{2}\right) q_{2} \\
\left(q_{2}^{2}-q_{1}^{2}\right) q_{2} & \left(q_{1}^{2}-q_{2}^{2}\right) q_{1}
\end{array}\right) \frac{\partial}{\partial x_{1}}\left(\begin{array}{l}
v_{1}-\beta q_{1} \\
v_{2}-\beta q_{2}
\end{array}\right) & \\
& +\left(\begin{array}{cc}
\left(q_{2}^{2}-q_{1}^{2}\right) q_{2} & \left(q_{1}^{2}-q_{2}^{2}\right) q_{1} \\
\left(q_{1}^{2}-q_{2}^{2}\right) q_{1} & \left(3 q_{1}^{2}+q_{2}^{2}\right) q_{2}
\end{array}\right) \frac{\partial}{\partial x_{2}}\left(\begin{array}{l}
v_{1}-\beta q_{1} \\
v_{2}-\beta q_{2}
\end{array}\right)=0
\end{aligned}
$$

This is easily seen to be a hyperbolic system whose characteristics make the angles $\pm \pi / 4$ with the direction of $\mathbf{q}$.

Since $\mathbf{q}$ is tangent to the die boundary, this boundary is not characteristic. Because the vector field $\mathbf{v}-\beta \mathbf{q}$ is a solution of the linear system (5.1) and vanishes on a noncharacteristic curve, it vanishes throughout the characteristic triangles determined by the die boundary. Thus the necessary condition (4.3) implies that $\mathbf{v}$ is an eigenvector of $D$ in these triangles. If $\lambda$ is the eigenvalue whose eigenvector is $\mathbf{v}$, then $\lambda=\mathbf{v} \cdot D \mathbf{v} /|\mathbf{v}|^{2}=\mathbf{v} \cdot \nabla \ln |\mathbf{v}|$. Since the speed is larger downstream than upstream, we must have $\lambda>0$. Thus, the necessary condition (4.3) implies that the flow is streamlined in the characteristic triangles determined by the upper and lower die boundaries. Because the flow is rigid outside these triangles, it is streamlined everywhere.

Richmond and Devenpeck [3] have, in fact, shown how to construct optimal twodimensional dies in the following manner. In two dimensions the equations (3.3) of streamlined flow reduce to

$$
\left(\begin{array}{cc}
\left(v_{1}^{2}+3 v_{2}^{2}\right) v_{1} & \left(v_{2}^{2}-v_{1}^{2}\right) v_{2} \\
\left(v_{2}^{2}-v_{1}^{2}\right) v_{2} & \left(v_{1}^{2}-v_{2}^{2}\right) v_{1}
\end{array}\right) \frac{\partial}{\partial x_{1}}\left(\begin{array}{c}
v_{1} \\
v_{2}
\end{array}\right)+\left(\begin{array}{cc}
\left(v_{2}^{2}-v_{1}^{2}\right) v_{2} & \left(v_{1}^{2}-v_{2}^{2}\right) v_{1} \\
\left(v_{1}^{2}-v_{2}^{2}\right) v_{1} & \left(3 v_{1}^{2}+v_{2}^{2}\right) v_{2}
\end{array}\right) \frac{\partial}{\partial x_{2}}\left(\begin{array}{c}
v_{1} \\
v_{2}
\end{array}\right)=0
$$

which is a hyperbolic system whose characteristics are at $45^{\circ}$ to $\mathbf{v}$.

Prescribe the restriction $\mathbf{v}\left(x^{1}, 0\right)$ of $\mathbf{v}$ along the line $x_{2}=0$, with $v_{2}\left(x^{1}, 0\right)=0$ so that the line is a streamline, and with $v_{1}\left(x^{1}, 0\right)$ a continuously differentiable function that is 
equal to the given inflow speed for $x_{1} \leq c$ and to the given outflow speed for $x_{1} \geq d$, and whose derivative is positive for $c<x_{1}<d$. Solve the system (5.2) both above and below the line with the prescribed initial values of $\mathbf{v}$ at $x_{2}=0$.

It is easy to see that the solution $\mathbf{v}$ of this initial value problem is equal to the prescribed constant horizontal upstream velocity to the left of the domain of influence of the interval $(c, d)$ of the $x^{1}$-axis, and to the downstream velocity to the right of this domain of influence.

One thus obtains the velocity field $\mathbf{v}$ of a streamlined flow. Any pair of streamlines of this flow gives an optimal die that converts an upstream flow with the prescribed speed and width to a downstream flow of the prescribed speed and the corresponding width.

Similar considerations apply for an axially symmetric flow. Here the velocity is constrained to be of the form

$$
\mathbf{v}=\left(\begin{array}{c}
u(r, z) \cos \theta \\
u(r, z) \sin \theta \\
w(r, z)
\end{array}\right)
$$

so that

$$
D=\left(\begin{array}{ccc}
\frac{\partial u}{\partial r} \cos ^{2} \theta+\frac{u}{r} \sin ^{2} \theta & \left(\frac{\partial u}{\partial r}-\frac{u}{r}\right) \sin \theta \cos \theta & \frac{1}{2}\left(\frac{\partial u}{\partial z}+\frac{\partial w}{\partial r}\right) \cos \theta \\
\left(\frac{\partial u}{\partial r}-\frac{u}{r}\right) \sin \theta \cos \theta & \frac{\partial u}{\partial r} \sin ^{2} \theta+\frac{u}{r} \cos ^{2} \theta & \frac{1}{2}\left(\frac{\partial u}{\partial z}+\frac{\partial w}{\partial r}\right) \sin \theta \\
\frac{1}{2}\left(\frac{\partial u}{\partial z}+\frac{\partial w}{\partial r}\right) \cos \theta & \frac{1}{2}\left(\frac{\partial u}{\partial z}+\frac{\partial w}{\partial r}\right) \sin \theta & \frac{\partial w}{\partial z}
\end{array}\right)
$$

This matrix has the eigenvalue $u / r$ with the eigenvector $(-\sin \theta, \cos \theta)$, which is perpendicular to $\mathbf{v}$. In addition, this matrix has two other eigenvalues, whose eigenvectors are of the same form as $\mathbf{v}$. Unless $u=0$, the other two eigenvalues are not negatives of each other.

If the eigenvector $\mathbf{q}$ that corresponds to the eigenvalue of largest magnitude is of the same form as $\mathbf{v}$ and tangent to the die boundary, then one can carry through the same argument as in the two-dimensional case to show that the necessary condition (4.3) implies that the flow is streamlined, so that the die is optimal.

In order to make these rather strong assumptions on $\mathbf{q}$ more plausible, we note that if the flow is smooth near the $z$-axis, then on the $z$-axis $D=\operatorname{diag}(\partial u / \partial r, \partial u / \partial r, \partial w / \partial z)$. We see from the divergence condition that the largest eigenvalue is $\partial w / \partial z=-2 \partial u / \partial r$. Thus on the $z$-axis $\mathbf{q}$ is in the $z$-direction. Moreover, because the velocity is larger downstream than upstream, $\lambda=\partial w / \partial z>0$ somewhere on the $z$-axis, and $u / r<0$ near this point. Thus if we only assume that $D$ never has the eigenvalue zero in the flow region, we conclude that $\lambda>0$ and $u<0$ everywhere. The latter condition states that the die is constricting.

Suppose now that the die boundary is connected, and that $\mathbf{q}$ is normal to the die boundary. Then $\mathbf{v}$ is an eigenvector of $D$, and because $\lambda>0$, the eigenvalue corresponding to $v$ must be negative. It follows that $\mathbf{v} \cdot \nabla|\mathbf{v}|^{2}=2 \mathbf{v} \cdot D \mathbf{v}<0$. This contradicts the fact that the downstream speed is greater than the upstream speed. Consequently, $\mathbf{q}$ must be tangent to the boundary, and the analogue of the two-dimensional proof shows that the flow is streamlined. We have thus shown that if an axially symmetric die is optimal and if the determinant of $D$ does not vanish in the nonrigid flow region, then the flow is streamlined. 
Richmond and Morrison [4] have shown how to construct a streamlined axially symmetric flow by solving the system of equations for $(u, w)$ with prescribed initial conditions on the axis of symmetry in a manner similar to the two-dimensional case. This again gives a flow whose streamlines can serve as optimal die boundaries.

6. The general die problem. In the general three-dimensional case the necessary condition (4.3) is far from implying that the flow is streamlined.

If the eigenvector $\mathbf{q}$ that corresponds to the maximal eigenvalue of $D$ is normal to the boundary, the flow cannot be streamlined. Even if we suppose that $\mathbf{q}$ is tangent to the boundary, the necessary condition (4.3) only implies that there is a scalar-valued function $\beta$ that is constant on $\mathbf{q}$-streamlines of the boundary such that the vector field $\mathbf{v}-\beta \mathbf{q}$ is perpendicular to $\mathbf{q}$ on the die boundary. This does not lead to the conclusion that $\mathbf{v}$ has the same direction as $\mathbf{q}$. Thus the necessary condition (4.3) does not seem to imply that the flow must be streamlined.

One could still hope to apply a construction like that of Richmond and Devenpeck in which one specifies a tangent vector field $\mathbf{v}$ on a surface that cuts the material lengthwise, with the prescribed constant velocities upstream and downstream, and then determines the flow from the streamlined flow equations (3.3).

The system (3.3) is hyperbolic, and its normal cone consists of the directions that are perpendicular to $\mathbf{v}$ and those that make a $45^{\circ}$ angle with $\mathbf{v}$. Therefore, the above initial surface is both characteristic and timelike. The latter means that the Cauchy problem with such an initial surface is not well posed. That is, it is not practical to try to compute a streamlined flow from data on such a surface. Moreover, even if one could compute such a flow, there is no reason to believe that the solution of the system (3.3) whose values on the initial surface have the prescribed constant velocities on the upstream and downstream parts of this surface will have these same constant velocities in any region off the surface, or that the prescribed cross sections are stream surfaces of this flow.

More generally, we observe that the upstream and downstream shapes of a die for twodimensional or axially symmetric flow are specified by a small number of parameters: the upstream and downstream velocities and the upstream width or radius. By contrast, in a general extrusion problem one must specify the upstream and downstream cross-sectional shapes, each of which involves infinitely many parameters.

These considerations make it quite unlikely that constructing special streamlined flows is useful in trying to optimize a general die.

Note added in proof. The author has, in fact, shown [J. Mech. Phys. Solids 45, 1275-1280 (1997)] that there are pairs of upstream and downstream cross sections for which there is no die which produces a streamlined flow.

On the other hand, the variational formula (4.2) indicates how to make small adjustments in the die boundary that decrease the rate of dissipation. One must, of course, take care to preserve the downstream cross section in this process. Such considerations may lead to a useful algorithm for approaching the optimal shape of a die.

Acknowledgments. This work was partially supported by a grant from the ALCOA Foundation. 


\section{ReFERENCES}

[1] R. Hill, Ideal forming operations for perfectly plastic solids, J. Mech. Phys. Solids 15, 223-227 (1967)

[2] G. Polya and M. Schiffer, Convexity of functionals by transplantation, J. d'Analyse Math. 3, 245346 (1954)

[3] O. Richmond and M. L. Devenpeck, A die profile for maximum efficiency in strip drawing, Proc. of the Fourth U. S. National Congress of Applied Mechanics, Vol. 2, pp. 1053-1057, 1962

[4] O. Richmond and H. L. Morrison, Streamlined wire drawing dies of minimum length, J. Mech. Phys. Solids 15, 195-203 (1967)

[5] H. F. Weinberger, Degenerate elliptic models for perfectly plastic flows, in Elliptic and Parabolic Problems, Pont-à Mousson, 1994, ed. Bandle, Bemelmans, Chipot, Paulin, and Shafrir, Pitman Research Notes in Mathematics 325, Longman, 1995, pp. 240-250 